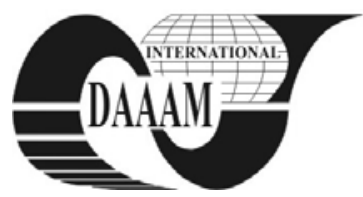

\title{
EFFECT OF TEMPERATURE INCREASING TO CURIE POINT ON THE MATERIAL COEFFICIENTS OF PZT CERAMICS
}

\author{
FIALKA, J[iri] \& UHER, M[iroslav]
}

\begin{abstract}
This paper deals with influence of temperature on the piezoelectric ceramics parameters. The properties of ceramics and its material coefficients are changing during the temperature increasing. The work is focused on the measurement of changes of piezoelectric coefficients after temperature increasing and subsequent decrease to room temperature. In applications where PZT ceramics is used, stable piezoelectric coefficients in wide-temperarure range are accentuated. In our research we measured the temperature dependencies from room temperature to temperature of Curie point.
\end{abstract}

Key words: PZT ceramics, resonance frequency, Curie temperature, coupling factor

\section{INTRODUCTION}

Each type of piezoelectric ceramics has its Curie point at different temperatures. This temperature is indicated by PZT ceramics manufacturers. However, in many cases the featured temperature may differ from the real value by tens of degrees. After determining of temperature dependency, it is possible to make description of permittivity, elastic and piezoelectric coefficients, evaluated from resonance frenquency and antirezonace frequency obtained from measurement close to Curie point. Measurements of temperature dependencies of PZT ceramics material coefficients near by or above Curie point are described in only a few publications. The majority of these papers are related to materials with different composition. In other cases the measurement is realized only for temperatures deep below Curie temperature. Within institutions dealing with this problem belong e.g. National Institute for material and physics in Bucurest (Miclea et al., 2006) or State University of Pensylvania (Hackenberger et al., 2004).

Material coefficients, which will be compared, are calculated indirectly from the size, density and resonance frequency and antiresonance frequency of PZT ceramics. Nowadays are resonant and antiresonance frequency most widely measured by the use of precision impedance analyzers. Among them belong to the most frequently used those produced by Agilent and Wayne Kerr, specifically the Agilent E4294A with a measuring range from $40 \mathrm{~Hz}$ to $120 \mathrm{MHz}$ and Wayne Kerr 65120B designed for frequencies from $20 \mathrm{~Hz}$ to $120 \mathrm{MHz}$. The main difference between these devices is the sensitivity and frequency range. For simultaneous measurement of impedance and phase can be used also cheaper RLC meters, such as HIOKI 3532 for frequencies from $42 \mathrm{~Hz}$ to $5 \mathrm{MHz}$ or Agilent E4980A, capable of measurements in range from $20 \mathrm{~Hz}$ to $2 \mathrm{MHz}$. These RLC meters have not got the suitable frequency range and accuracy. Data processing of frequency dependence is controlled by the programs such as LabVIEW produced by National Instruments or VEE by Agilent.

For measurement of frequency dependencies of impendance and phase to higher frequencies, it is necessary to use special measuring pliers, e.g. the "Tweezers contact test fixture 16334A" by Agilent or "Pincher Probe 9143" by HIOKI.

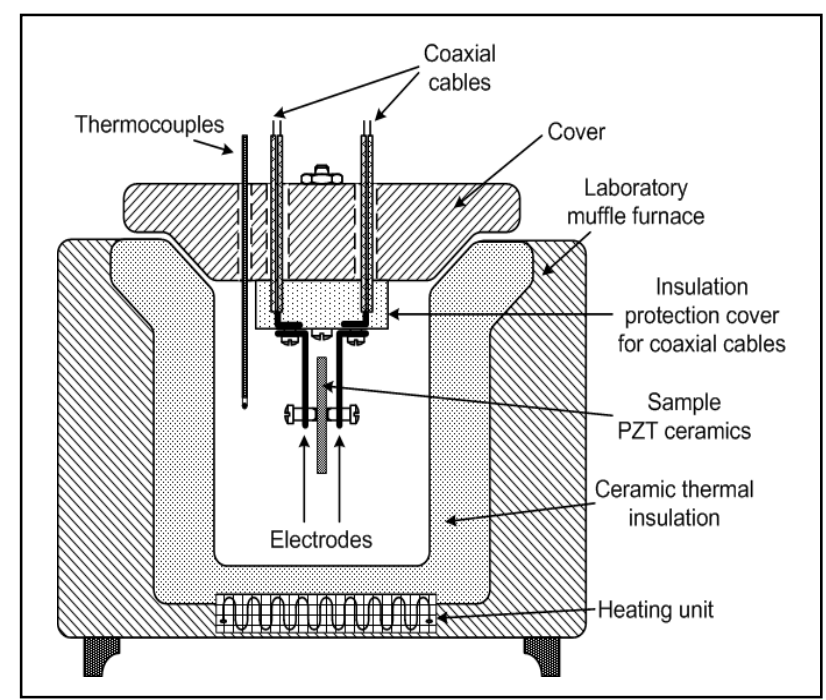

Fig. 1. Experimental muffle furnace for measuring of the thermal response PZT ceramics

Was it necessary to produce special tools for measuring at high temperatures? As majority of measuring cables have limited temperature range, in most cases it is around $100^{\circ} \mathrm{C}$, we needed to produce a special clam, capable of high temperature measurements. This measuring clam so-called „Tweezer“, was directly built in the heating furnace cover. The constructional scheme of the measuring tweezer inserted inside the muffle furnace is shown in the Figure 1.

This method enables the use of conventional coaxial cables routed through the cover directly to the measuring electrodes. Over the top cover there is an inserted thermocoupler of appropriate type, which is used for temperature measurements. The termocoupler is connected to the measuring device by a shielded cable.

Instrument electrode „Tweezer“, made of rustfree sheet plate with screws to fix the piezoelectric element, is attached to a ceramic back-plate. Free space between cables and cover inside ceramic back-plate is filled with soft sand. Laboratory muffle furnace is a combination of an inside isolating ceramics and thermo-insulating outer case. Heating of the oven is provided with the heater placed below the ceramic lining.

\section{MEASUREMENTS}

\subsection{Measured samples}

Before making the numerical calculation of the piezoelectric constant, it was necessary to produce samples. The dimensions and shapes of these samples have to correspond to the IEEE standard of piezoelectricity CEI/IEC 60483: 1976 (CEI/IEC 60483, 1976). For this experiment we have choosen a "soft type" piezoelectric materials PCM51, dimensions are shown in Tab 1 . The value of the Curie point for the material PCM51 is reported by producer at $360^{\circ} \mathrm{C}$. 


\begin{tabular}{|c|c|c|c|c|}
\hline $\begin{array}{c}\text { Sample and } \\
\text { dimension }\end{array}$ & $\varnothing d$-diameter & h-thickness & l-length & w-width \\
\cline { 2 - 5 } & {$[\mathrm{mm}]$} & {$[\mathrm{mm}]$} & {$[\mathrm{mm}]$} & {$[\mathrm{mm}]$} \\
\hline Thin disc & 30,0 & 2,0 & - & - \\
\hline Thin plate & - & 2,0 & 4,0 & 25,0 \\
\hline Cylinder & 3,5 & 20,1 & - & - \\
\hline
\end{tabular}

Tab. 1. Real dimensions of samples for complete set of piezoelectric material calculation

\subsection{Frequency measurement method}

LCR-meter HIOKI 3532 capable of measuring from $20 \mathrm{~Hz}$ to $2 \mathrm{MHz}$ was used for reading of values. This LCR-meter was connected to desktop computer by a USB port. For configuration, data download and showing of measured of dates was created an application in programme LabVIEW. The advantage of this construction was the possibility to show impedance and phase characteristics directly, and fast revision of the measured dates.

Electromechanical piezoelectric coefficient was chosen to verify the effect of temperature on material factors. It could be expressed as the ratio of used electric energy to transducer to the mechanical strain energy (Waanders et al., 1991).

Equation (1) is used for calculating of electromechanical coupling factor $k_{31}$ for transverse modes in ceramic plate:

$$
k_{31}^{2}=\frac{\frac{\pi}{2} \cdot \frac{f a}{f_{r}} \cdot \tan \left(\frac{\pi}{2} \cdot \frac{f a-f_{r}}{f_{r}}\right)}{1+\frac{\pi}{2} \cdot \frac{f a}{f_{r}} \cdot \tan \left(\frac{\pi}{2} \cdot \frac{f a-f_{r}}{f_{r}}\right)}
$$

Equation (2) is used for determining of electromechanical coupling factor $k_{33}$ for longitudinal modes in ceramic rod and electromechanical coupling factor $k_{t}$ for thickness modes in ceramic disk:

$$
k_{33}^{2} \cong k_{t}^{2}=\frac{\pi}{2} \cdot \frac{f_{a}}{f_{r}} \cdot \tan \left(\frac{\pi}{2} \cdot \frac{f_{a-} f_{r}}{f_{a}}\right)
$$

Finally, equation (3) expresses relation for calculating of electromechanical coupling factor $k_{p}$ for planar modes in ceramic disk:

$$
k_{p}^{2}=2,51 \cdot \frac{\left(f_{a-}-f_{r}\right)}{f_{r}}-\left(\frac{f_{a-}-f_{r}}{f_{r}}\right)^{2}
$$

Where $k_{31}$ is coupling factor for transverse mode (-), $k_{33}$ is coupling factor for longitudinal modes (-), $k_{p}$ is coupling factor for planar mode (-), $k_{t}$ is coupling factor for thickness mode (-), $f_{r}$ is resonance frequency $(\mathrm{Hz})$ and $f_{a}$ is antiresonance frequency (Hz) (APC International, 2002).

Figure 2 shows dependency of electromechanical coupling factor on temperature during heating of the sample. On the other hand, Figure 3 shows temperature dependency of electromechanical coupling factor after temperature drop to room temperature $25^{\circ} \mathrm{C}$.

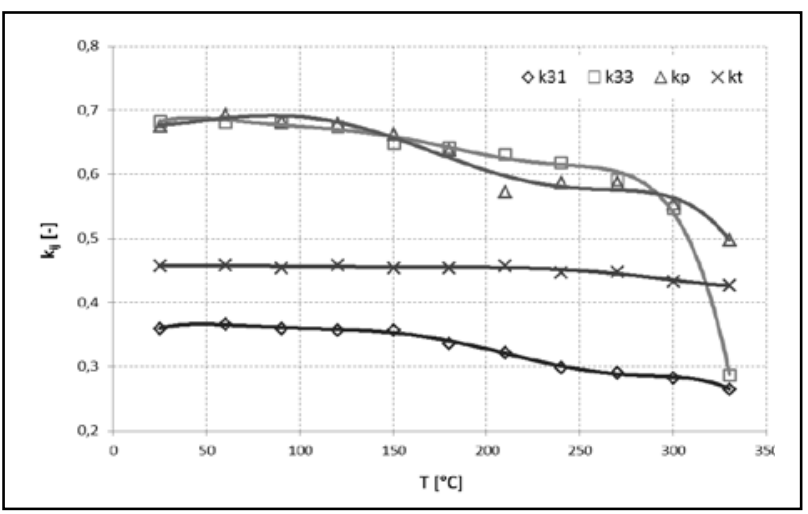

Fig. 2. The temperature dependence of the electromechanical coupling factor $\mathrm{k}_{33}, \mathrm{k}_{31}, \mathrm{k}_{\mathrm{p}}$ and $\mathrm{k}_{\mathrm{t}}$ between $25^{\circ} \mathrm{C}$ and $330{ }^{\circ} \mathrm{C}$ for temperature rise

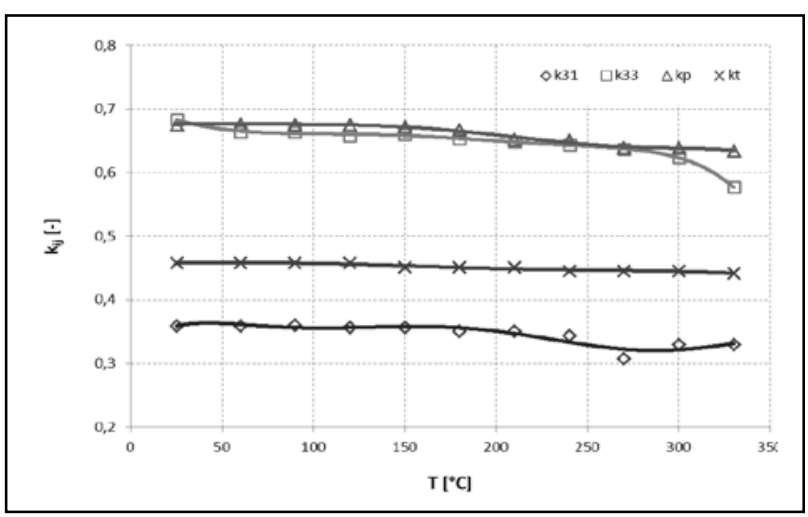

Fig. 3. The temperature dependence of the electromechanical coupling factor $\mathrm{k}_{33}, \mathrm{k}_{31}, \mathrm{k}_{\mathrm{p}}$ and $\mathrm{k}_{\mathrm{t}}$ between $25^{\circ} \mathrm{C}$ and $330{ }^{\circ} \mathrm{C}-$ after temperature drop

\section{CONCLUSION}

The original plan on this measuring was to investigate the behaviour of the PZT ceramics at increasing temperature, to calculate important piezoelectric material constant and to show their temperature dependencies. Short-term influence of the ambient temperature on PZT ceramics will not damage this material and will not change its parameters. If the temperature approaches the Curie point, the piezoelectric constants begin significantly to change its value. After termination of the actuation of high temperature, the material is already not capable of reaching to its initial values. That is why it is necessary to take into account this limitation when is designing the apparatus using piezoelectric.

\section{ACKNOWLEDGEMENTS}

This paper was supported in part by the Ministry of Education, Youth and Sports of the Czech Republic (Research Intent MSM0021630529 Intelligent systems in automation), Grant Agency of the Czech Republic (102/09/H082 SYNERGY - Sensors and Intelligent Sensor Systems) and by grant "Supporting Research of Modern Methods and Approaches in Automation" from the Internal Grant Agency of Brno University of Technology (grant No. FEKT-S-11-6).

\section{REFERENCES}

Hackenberger, W. etal. (2004). High Temperature Electrostrictive Ceramics for a Venus Ultrasonic Rock Sampling Tool. 14th IEEE International Symposium on Applications of Ferroelectrics - ISAF-04, pg. 130-133. ISBN 0-78038410-5

Miclea, C. etal. (2006). Temperature Dependence of the Main Piezoelectric Parameters of aNb-Li Doped PZT Ceramic. International Semiconductor Conference 2006. BucharestMagurele: National Inst. for Mater. Phys., vol. 2, pp. 279282. ISBN 1-4244-0109-7

Waanders, J.B. (1991). Piezoelectric Ceramics: Properties and Applications, Philips Components, ISBN 9398 651-80011, Eindhoven - The Netherlands

*** (2002) APC International - Piezoelectric Theory and applications, Piezoelectric Ceramics: Principles and Applications, American Piezo Ceramics Inc., pg. 112, ISBN 0-9718744-0-9

*** (1976) International Standard CEI/IEC 60483:1976, Guide to dynamic measurements of piezoelectric ceramics with high electromechanical coupling, accessed on: 1976-01 